\title{
Robust Adaptive Nonlinear Dynamic Inversion Control for an Air-breathing Hypersonic Vehicle
}

\author{
Guanghui Wu , Xiuyun Meng and Jie Wang \\ School of Aerospace Engineering, Beijing Institute of Technology, 100081, Beijing, China
}

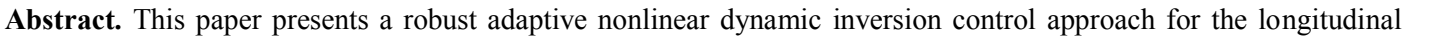

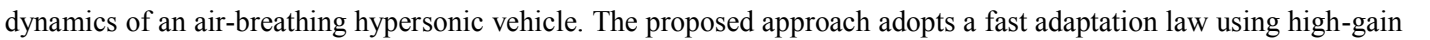

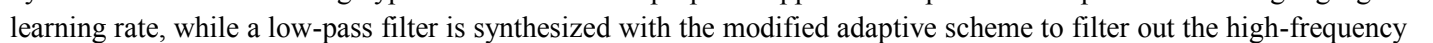

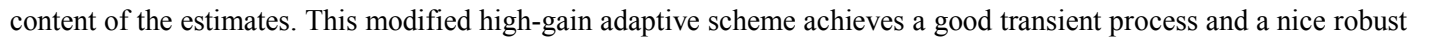

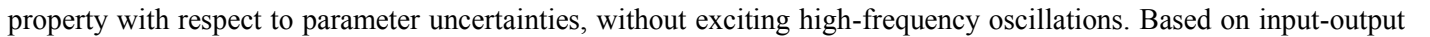

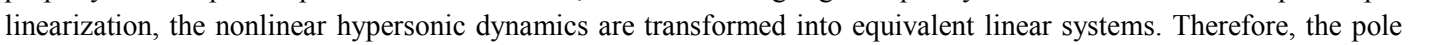

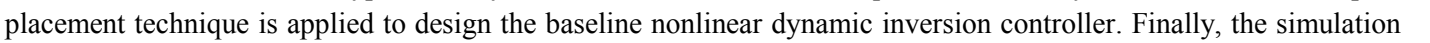

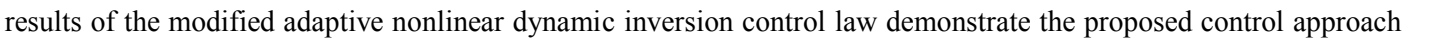

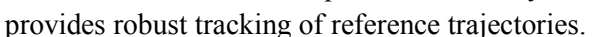

\section{Introduction}

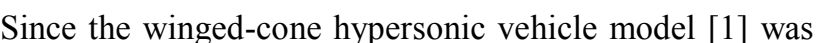
பயாயாமாம

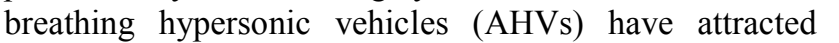

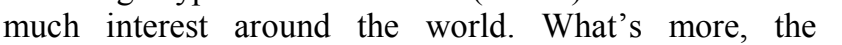
पण

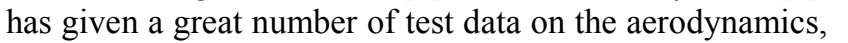

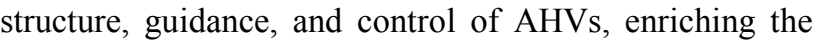
एण

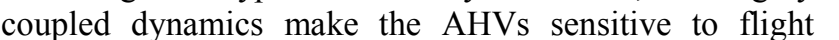

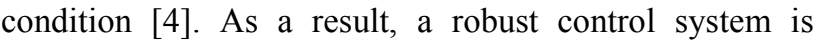

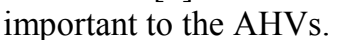

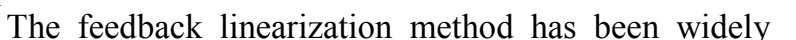
एण1

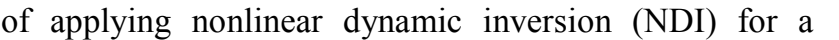
பण

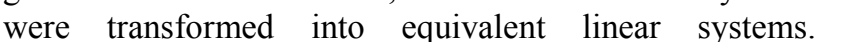

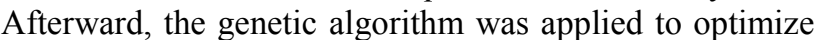

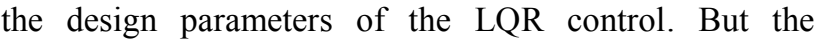

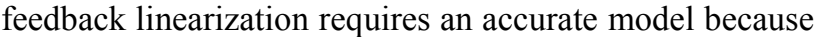

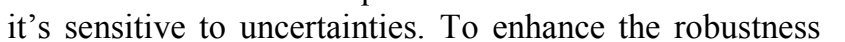

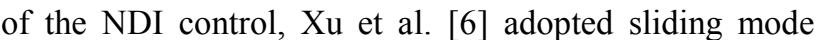

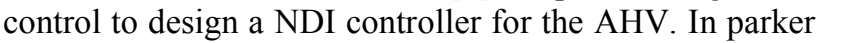

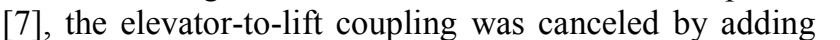

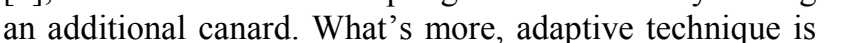

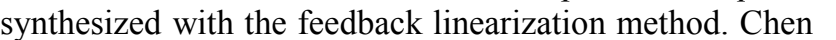

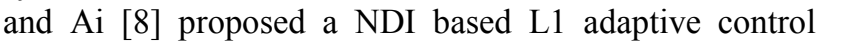

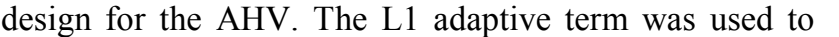

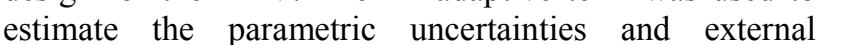

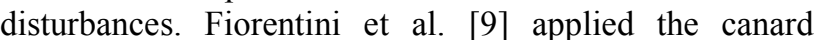

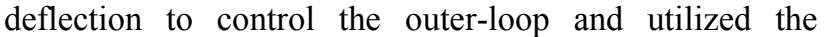

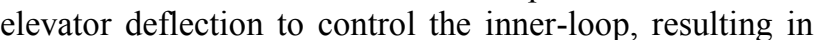

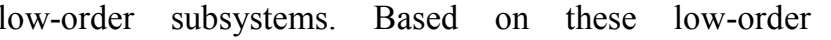

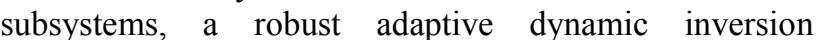

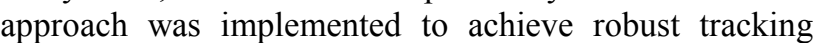
पाणाणमाणा

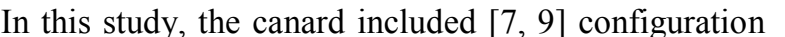

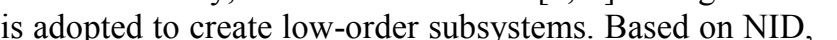

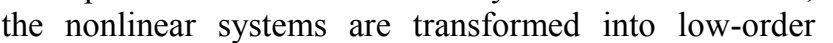

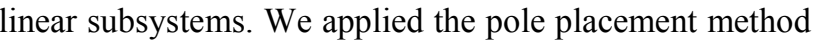

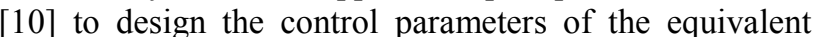

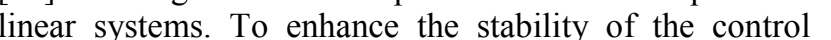

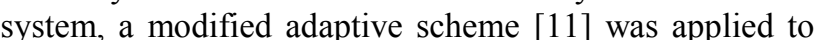

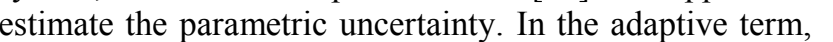

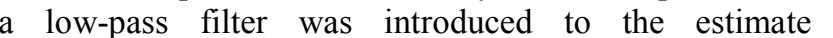

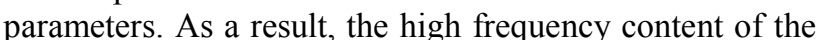

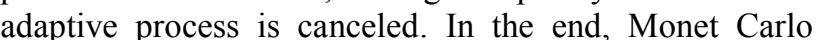

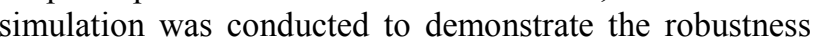

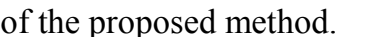

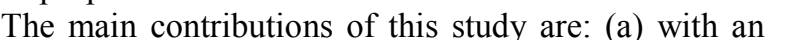

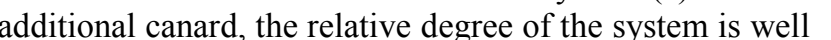

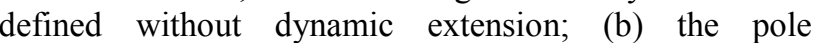

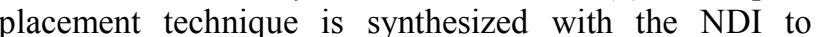

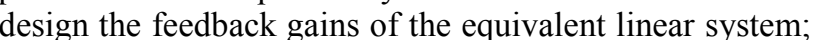

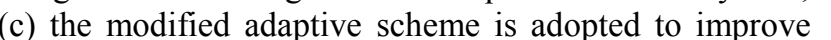

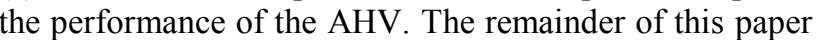

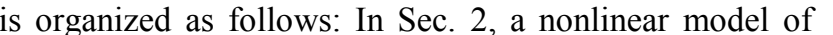

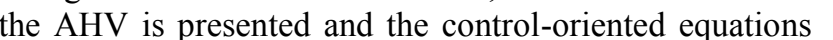

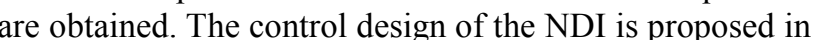

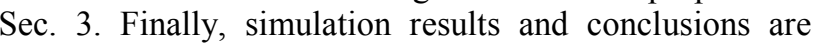

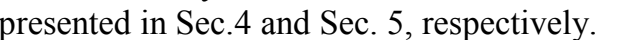

\section{Model description}




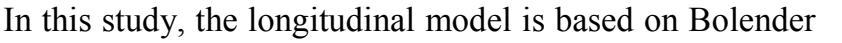

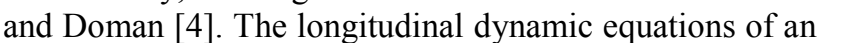

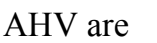

$$
\begin{gathered}
\dot{V}=(T \square \text { a }-D) \square m-g \square \square \\
\square \quad \dot{h}=V \square \square \gamma \square \\
\square \quad \dot{\gamma}=(L+T \square \square) \square(m V)-g \square \square \gamma \square \\
\square \quad \dot{\theta}=q \square \\
\square \quad \dot{q}=M \square I_{y y} \square
\end{gathered}
$$

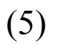

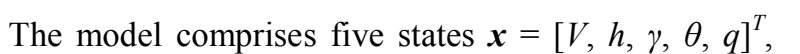

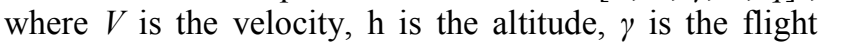

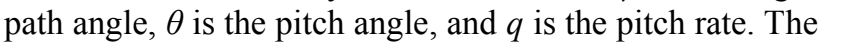

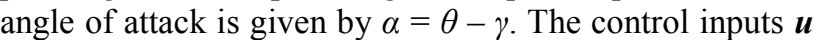

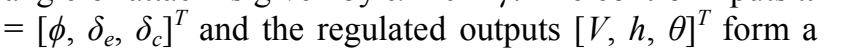

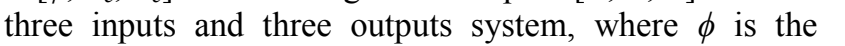

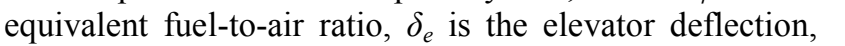

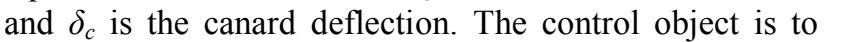

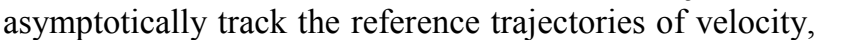

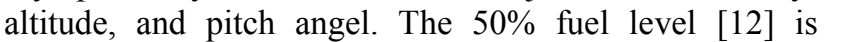

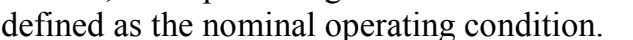

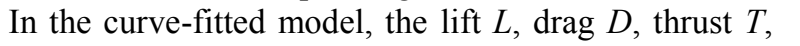

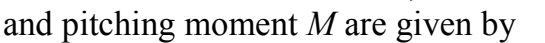

$$
\begin{aligned}
& T \approx \bar{q} S\left[C_{T \phi}(\alpha) \phi+C_{T}(\alpha)\right] \\
& L \approx \bar{q} S C_{L}(\alpha \boldsymbol{\delta}) \\
& D \approx \bar{q} S C_{D}(\alpha \boldsymbol{\delta}) \\
& M \approx z_{T} T+\bar{q} S \bar{c} C_{M}(\alpha \boldsymbol{\delta})
\end{aligned}
$$

$\square \square$

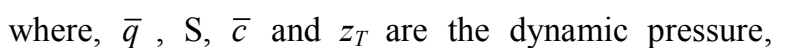

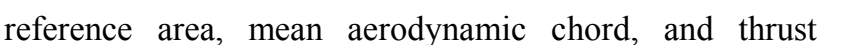

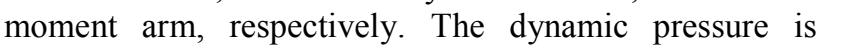

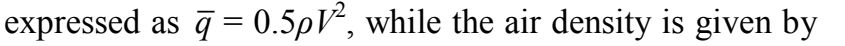

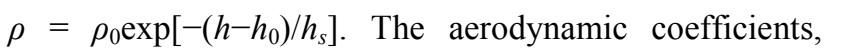

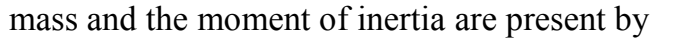

$$
\begin{aligned}
& \boldsymbol{\delta}=\left[\delta_{e} \delta_{c}\right]^{T} \\
& C_{T \phi \phi}(\alpha)=v_{\square} C_{T}^{\phi \alpha} \alpha+v_{\square} C_{T}^{\phi \alpha} \alpha+v_{\square} C_{T}^{\phi \alpha} \alpha+v_{\square} C_{T}^{\phi} \\
& C_{T}(\alpha)=v_{\square} C_{T}^{\square}+v_{\square} C_{T}^{\square}+v_{\square} C_{T}^{\square} \alpha+v_{\square} C_{T}^{\square} \\
& C_{L}(\alpha \delta)=v_{\square} C_{L}^{\alpha} \alpha+v_{\square} C_{L}^{\delta_{e}} \delta_{e}+v_{\square} C_{L}^{\delta_{c}} \delta_{c}+v_{\square} C_{L}^{\square} \\
& C_{D}(\alpha \delta)=v_{\square} C_{D}^{\alpha} \alpha+v_{\square} C_{D}^{\alpha} \alpha+v_{\square} C_{D}^{\square} \\
& C_{M}(\alpha \boldsymbol{\delta})=v_{\square} C_{M}^{\alpha} \alpha+v_{\square} C_{M}^{\alpha} \alpha+v_{\square} C_{M}^{\delta_{e}} \delta_{e}+ \\
& \quad v_{\square} C_{M}^{\delta_{c}} \delta_{c}+v_{\square} C_{M}^{\square} \\
& m=v_{\square} m_{\square} \\
& I_{y y}=v_{\square} I_{y y \square}
\end{aligned}
$$

\begin{tabular}{|c|c|c|c|}
\hline 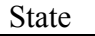 & $\square \square \square \square$ & 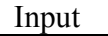 & 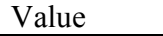 \\
\hline V & 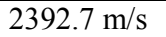 & $\phi$ & 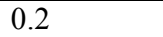 \\
\hline$h$ & $\square 11 \| \square \square$ & $\delta_{e}$ & पणापाणा \\
\hline$\gamma$ & 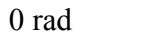 & $\delta_{c} \square$ & $-\square\|1\| \|$ \\
\hline$\theta$ & 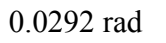 & $\square$ & $\square$ \\
\hline$Q$ & 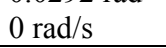 & $\square$ & $\square$ \\
\hline
\end{tabular}

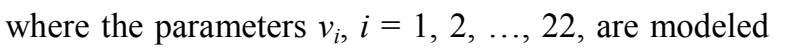

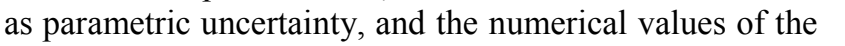

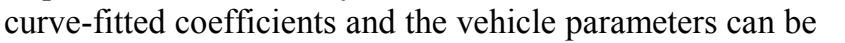

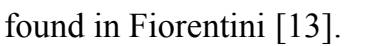

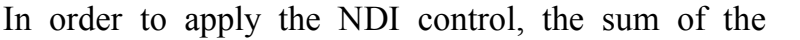

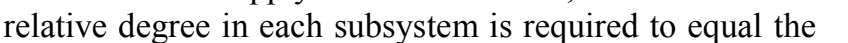

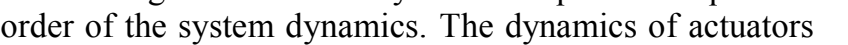
पाm

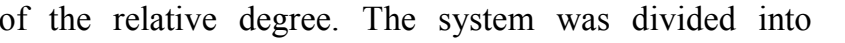
ए।

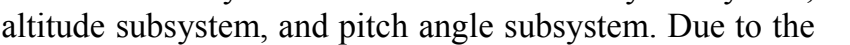

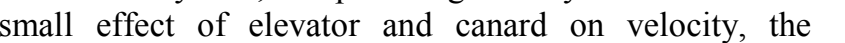

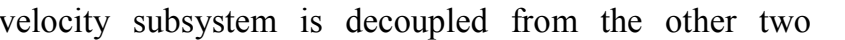

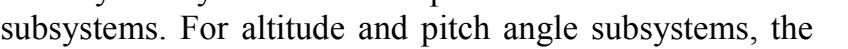

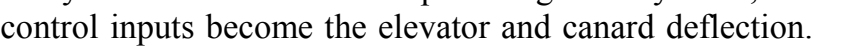

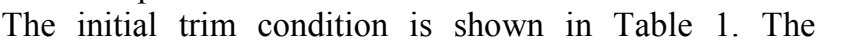

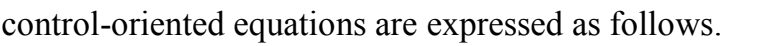

Table 1.

$\square$

$$
\dot{V}=\theta_{\square}^{T} \varphi_{\square}+\theta_{\square}^{T} \varphi \phi-g \square \square \gamma \square
$$

$$
\begin{aligned}
& \dot{h}=V \square \square \gamma \\
& \ddot{h}=\theta_{\square}^{T} \varphi_{\square}+\theta_{\square}^{T} \varphi_{\square} \delta-g \\
& \dot{\theta}=q \\
& \ddot{\theta}=\theta_{\square}^{T} \varphi_{\square}+\theta_{\square}^{T} \varphi_{\square}
\end{aligned}
$$

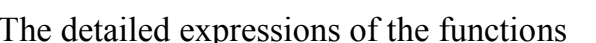

$$
\begin{aligned}
& \theta_{\square}=\left(\square \square v_{\square}\right)\left[v_{\square} \vee_{\square} \vee_{\square} \boldsymbol{v}_{\square} \boldsymbol{v}_{\square} \vee_{\square} \boldsymbol{v}_{\square}\right]^{T} \\
& \varphi=\left(\bar{q} S \square m_{\square}\right) \alpha^{\square} \square \alpha C_{T}^{\square} \alpha^{\square} \square \alpha C_{T}^{\square} \alpha \square \alpha \alpha C_{T}^{\square} \square \\
& \left.\square \quad \square \square \alpha C_{T}^{\square} \square \alpha C_{D}^{\alpha \square} \square \alpha C_{D}^{\alpha} \square C_{D}^{\square}\right]^{T} \\
& \theta_{\square}=\left(\square \square \boldsymbol{v}_{\square}\right)\left[v_{\square} \boldsymbol{v}_{\square} \boldsymbol{v}_{\square} \boldsymbol{v}_{\square}\right]^{T} \\
& \varphi_{\square}=\left(\bar{q} S \square \alpha \square m_{\square}\right)\left[\alpha C_{T}^{\phi \alpha} \quad \alpha C_{T}^{\phi \alpha} \square \alpha C_{T}^{\phi \alpha} C_{T}^{\phi}\right]^{T}
\end{aligned}
$$

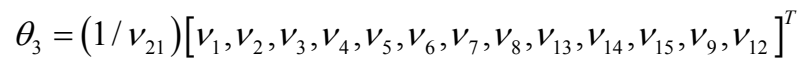

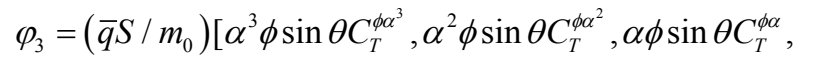

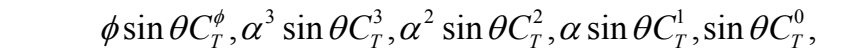
$-\alpha \square \square \gamma C_{D}^{\alpha} \square \alpha \square \square \gamma C_{D}^{\alpha} \square \square \square \gamma C_{D}^{\square} \alpha \square \gamma C_{L}^{\alpha} \square$ $\square \gamma C_{L}^{\square} T$

$$
\begin{aligned}
& \theta_{\square}=\left(\square \square v_{\square}\right)\left[v_{\square} v_{\square}\right]^{T} \\
& \varphi_{\square}=\left(\bar{q} S \square \square \gamma \square m_{\square}\right) \operatorname{diag}\left(C_{L}^{\delta_{e}}\left[C_{L}^{\delta_{c}}\right)\right.
\end{aligned}
$$

$$
\begin{aligned}
& \theta_{\square}=\left(\square \square \boldsymbol{v}_{\square}\right)\left[\boldsymbol{v}_{\square} \boldsymbol{v}_{\square} \boldsymbol{v}_{\square} \boldsymbol{v}_{\square} \boldsymbol{v}_{\square} \boldsymbol{v}_{\square} \boldsymbol{v}_{\square} \boldsymbol{v}_{\square} \boldsymbol{v}_{\square} \nabla_{\square} \boldsymbol{v}_{\square}\right]^{T} \\
& \varphi_{\square}=\left(\bar{q} S \square I_{y y \square}\right) z_{T} \alpha \phi C_{T}^{\phi \alpha} \square z_{T} \alpha \phi C_{T}^{\phi \alpha \square} z_{T} \alpha \phi C_{T}^{\phi \alpha} \square \\
& z_{T} \phi C_{T}^{\phi} \Xi_{T} \alpha C_{T}^{\square} z_{T} \alpha C_{T}^{\square} z_{T} \alpha C_{T}^{\square} z_{T} C_{T}^{\square} \bar{c} \alpha C_{M}^{\alpha \square} \square \\
& \bar{c} \alpha C_{M}^{\alpha} \bar{c} C_{M}^{\square}
\end{aligned}
$$




$$
\begin{aligned}
& \theta_{\square}=\left(\square \boldsymbol{v}_{\square}\right)\left[v_{\square} \vee_{\square}\right]^{T} \\
& \varphi_{\square}=\left(\bar{q} S \bar{c} \square_{y y \square}\right) \operatorname{diag}\left(C_{M}^{\delta_{e}}\left(C_{M}^{\delta_{c}}\right)\right.
\end{aligned}
$$

\section{Control design}

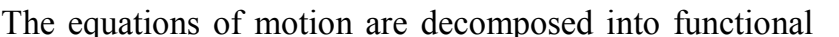

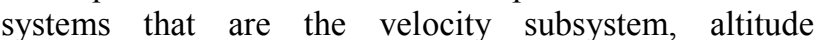

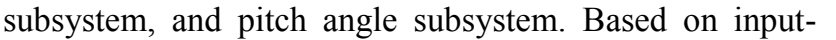

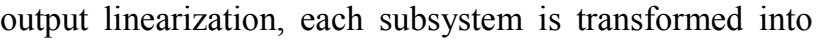

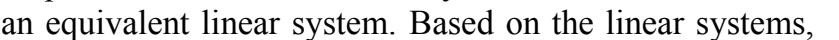

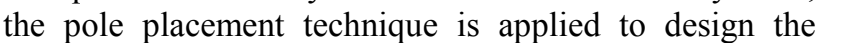

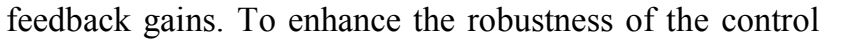

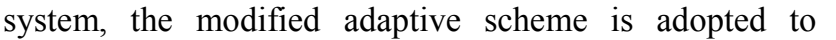

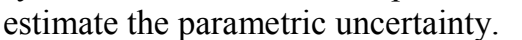

\subsection{NDI control for the velocity subsystem}

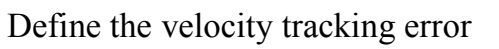

$$
\tilde{V}=V-V_{\text {ref }} \mathbb{W}
$$

एा1ए

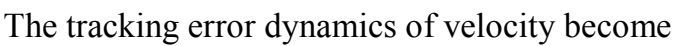

$$
\dot{\tilde{V}}=\theta_{\square}^{T} \varphi_{\square}+\theta_{\square}^{T} \varphi \phi-g \square \square \gamma-\dot{V}_{\text {ref }} \square
$$

एा11

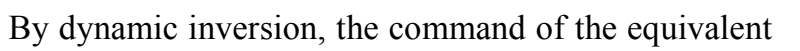

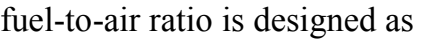

$$
\square \quad \phi=\left(-k_{P V} \tilde{V}-\hat{\theta}_{\square}^{T} \varphi+g \square \gamma+\dot{V}_{r e f}\right)\left(\hat{\theta}_{\square}^{T} \varphi_{\square}\right) \square \square \square \square
$$

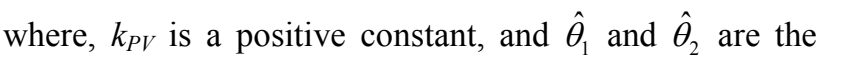

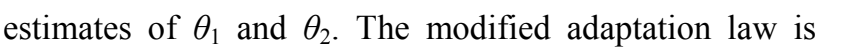

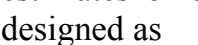

$$
\begin{gathered}
\dot{\hat{\theta}}_{\square}=\Gamma_{\square} \square \square\left(\tilde{V} \varphi_{\square}-\sigma_{\square}\left(\hat{\theta}_{\square}-\hat{\theta}_{\square f}\right)\right) \square \\
\dot{\hat{\theta}}_{\square}=\Gamma_{\square} \square \square\left(\tilde{V} \varphi_{\square} \phi-\sigma_{\square}\left(\hat{\theta}_{\square}-\hat{\theta}_{\square f}\right)\right) \square \square \square \square
\end{gathered}
$$

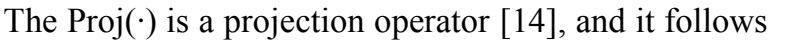

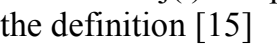

$$
-\tilde{\theta}_{i}(\square \square(y)-y) \leq \square i=\square \| \square \square \square \square \square
$$

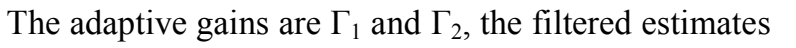

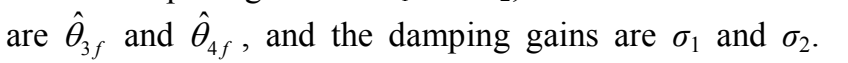

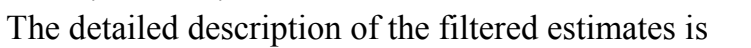

$$
\begin{aligned}
& \dot{\hat{\theta}}_{\square f}=\Gamma_{\square f}\left(\hat{\theta}_{\square}-\hat{\theta}_{\square f}\right) \square \\
& \dot{\hat{\theta}}_{\square f}=\Gamma_{\square f}\left(\hat{\theta}_{\square}-\hat{\theta}_{\square f}\right) \square
\end{aligned}
$$

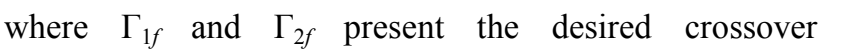

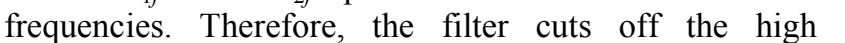

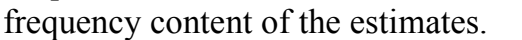

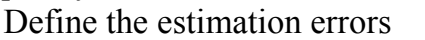

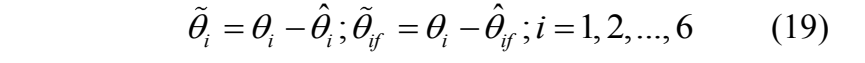

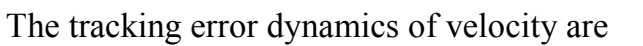

$$
\dot{\tilde{V}}=-k_{P V} \tilde{V}+\tilde{\theta}_{\square}^{T} \varphi+\tilde{\theta}_{\square}^{T} \varphi \phi \square
$$

$\square+11$

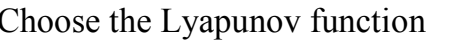

$$
\begin{gathered}
W_{V}=\tilde{V}^{\square} \square+\tilde{\theta}_{\square}^{T} \Gamma_{\square}^{-} \tilde{\theta} \square+\left(\sigma_{\square} \square\right) \tilde{\theta}_{\square f}^{T} \Gamma_{\square f}^{-\square} \tilde{\theta}_{\square f}+ \\
\tilde{\theta}_{\square}^{T} \Gamma_{\square}^{-} \tilde{\theta}_{\square} \square+\left(\sigma_{\square} \square\right) \tilde{\theta}_{\square f}^{T} \Gamma_{\square f}^{-\square} \tilde{\theta}_{\square f}
\end{gathered}
$$
पाIII

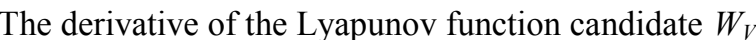

$$
\begin{aligned}
& \dot{W}_{V}=-k_{P V} \tilde{V}^{\square}+\tilde{V} \tilde{\theta}_{\square}^{T} \varphi+\tilde{V} \tilde{\theta}_{\square}^{T} \varphi \phi-\tilde{\theta}_{\square}^{T} \Gamma_{\square}^{-} \dot{\hat{\theta}}-\sigma \tilde{\theta}_{f f}^{T} \Gamma_{\square f}^{-} \dot{\hat{\theta}}_{f f}- \\
& \tilde{\theta}_{\square}^{T} \Gamma_{\square}^{-} \dot{\hat{\theta}}_{\square}-\sigma \tilde{\theta}_{\square f}^{T} \Gamma_{\square f}^{-0} \dot{\hat{\theta}}_{\square f} \\
& =-k_{P V} \tilde{V}^{\square}+\tilde{V} \tilde{\theta}_{\square}^{T} \varphi_{\square}+\tilde{V} \tilde{\theta}_{\square}^{T} \varphi \phi-\tilde{\theta}_{\square}^{T} \square \square\left(\tilde{V} \varphi_{\square}-\sigma\left(\tilde{\theta}_{f f}-\tilde{\theta}\right)\right)- \\
& \sigma \tilde{\theta}_{f f}^{T}\left(\tilde{\theta}_{f f}-\tilde{\theta}\right)-\tilde{\theta}_{\square}^{T} \square\left(\tilde{V} \varphi \phi-\sigma\left(\tilde{\theta}_{\square f}-\tilde{\theta}\right)\right)- \\
& \sigma \tilde{\theta}_{\square f}^{T}\left(\tilde{\theta}_{\square f}-\tilde{\theta}_{\square}\right) \\
& =-k_{P V} \tilde{V}^{\square}+\tilde{V} \tilde{\theta}_{\square}^{T} \varphi-\sigma \tilde{\theta}_{\square}^{T}\left(\tilde{\theta}_{f f}-\tilde{\theta}\right)-\sigma\left(\tilde{\theta}_{f f}-\tilde{\theta}\right)^{T}\left(\tilde{\theta}_{f f}-\tilde{\theta}\right)- \\
& \tilde{\theta}^{T} \square \square\left(\tilde{V} \varphi-\sigma\left(\tilde{\theta}_{\text {If }}-\tilde{\theta}\right)\right)+\tilde{V} \tilde{\theta}_{\square}^{T} \varphi \phi-\sigma \tilde{\theta}_{\square}^{T}\left(\tilde{\theta}_{\square f}-\tilde{\theta}_{\square}\right)- \\
& \tilde{\theta}_{\square}^{T} \square\left(\tilde{V} \varphi \phi-\sigma\left(\tilde{\theta}_{\square f}-\tilde{\theta}_{\square}\right)\right)-\sigma_{\square}\left(\tilde{\theta}_{\square f}-\tilde{\theta}_{\square}\right)^{T}\left(\tilde{\theta}_{\square f}-\tilde{\theta}_{\square}\right) \\
& \leq-k_{P V} \tilde{V}^{\square}-\sigma\left(\tilde{\theta}_{f f}-\tilde{\theta}\right)^{T}\left(\tilde{\theta}_{\square f}-\tilde{\theta}\right)-\sigma_{\square}\left(\tilde{\theta}_{\square f}-\tilde{\theta}_{\square}\right)^{T}\left(\tilde{\theta}_{\square f}-\tilde{\theta}_{\square}\right) \\
& \leq \square
\end{aligned}
$$

\subsection{NDI control for the altitude and pitch subsystems}

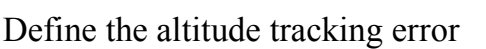

$$
\tilde{h}=h-h_{r e f} \dot{\tilde{h}}=V \square \square \gamma-\dot{h}_{r e f} \square
$$

$\mathbb{1 1 0}$

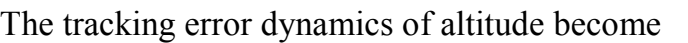

$$
\ddot{\widetilde{h}}=\theta_{\square}^{T} \varphi+\theta_{\square}^{T} \varphi \delta-g-\ddot{h}_{r e f} \square
$$

एाI

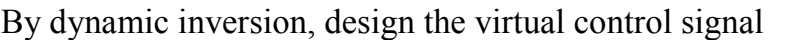

$$
\square \quad u_{h}=\hat{\theta}_{\square}^{T} \varphi \delta=-k_{P h} \tilde{h}-k_{D h} \dot{\tilde{h}}-\hat{\theta}_{\square}^{T} \varphi_{\square}+g+\ddot{h}_{r e f} \square \square \square
$$

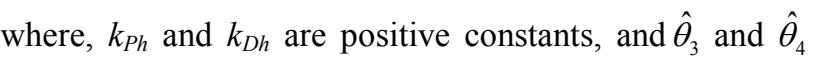

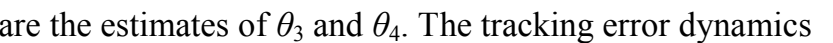
$\square ण \square \square$

$\square \quad\left[\begin{array}{c}\dot{\tilde{h}} \\ \ddot{\tilde{h}}\end{array}\right]=\underbrace{\left[\begin{array}{cc}\square & \square \\ -k_{P h} & -k_{D h}\end{array}\right]}_{A_{h}}\left[\begin{array}{c}\tilde{h} \\ \dot{\tilde{h}}\end{array}\right]+\left[\begin{array}{c}\square \\ \square\end{array}\right]\left(\tilde{\theta}_{b_{h}}^{T} \varphi_{\square}+\tilde{\theta}_{\square}^{T} \varphi \delta\right) \square \square \square$

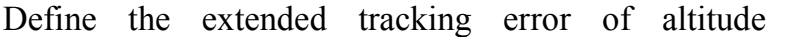

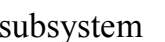

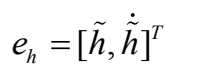

एा1ए 


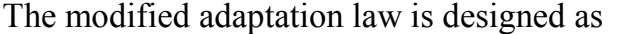

$$
\begin{aligned}
& \dot{\hat{\theta}}_{\square}=\Gamma_{\square} \square \square\left(\varphi e_{h}^{T} P b-\sigma_{\square}\left(\hat{\theta}_{\square}-\hat{\theta}_{\square f}\right)\right) \square \square \square \square \\
& \dot{\hat{\theta}}_{\square}=\Gamma_{\square} \square\left(\varphi \delta e_{h}^{T} P b-\sigma_{\square}\left(\hat{\theta}_{\square}-\hat{\theta}_{\square f}\right)\right) \square \square \square \square
\end{aligned}
$$

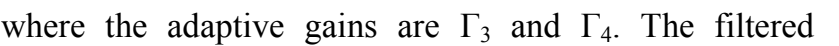

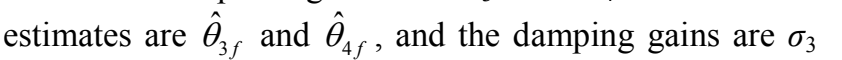

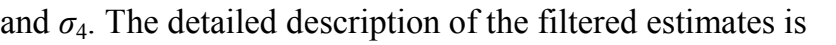

$$
\begin{aligned}
& \dot{\hat{\theta}}_{\square f}=\Gamma_{\square f}\left(\hat{\theta}_{\square}-\hat{\theta}_{\square f}\right) \square \\
& \dot{\hat{\theta}}_{\square f}=\Gamma_{\square f}\left(\hat{\theta}_{\square}-\hat{\theta}_{\square f}\right) \square
\end{aligned}
$$

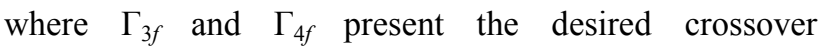

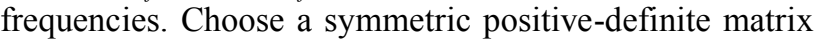

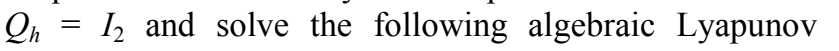
पाणाणाण

$$
P_{h} A_{h}+A_{h}^{T} P_{h}=-Q_{h} \square
$$

एा1ए

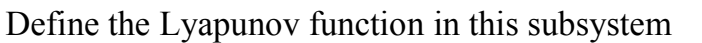

$$
\begin{gathered}
W_{h}=e_{h}^{T} P_{h} e_{h} \square+\tilde{\theta}_{\square}^{T} \Gamma_{\square}^{-\square} \tilde{\theta}_{\square} \square+\left(\sigma_{\square} \square\right) \tilde{\theta}_{\square f}^{T} \Gamma_{\square f}^{-\square} \tilde{\theta}_{\square f}+ \\
\tilde{\theta}_{\square}^{T} \Gamma_{\square}^{-} \tilde{\theta}_{\square} \square+\left(\sigma_{\square} \square\right) \tilde{\theta}_{\square f}^{T} \Gamma_{\square f}^{-\square} \tilde{\theta}_{\square f}
\end{gathered}
$$

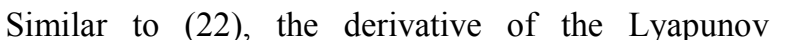

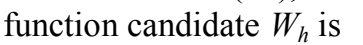

$$
\begin{aligned}
\dot{W}_{h}= & -e_{h}^{T} Q_{h} e_{h} \square+e_{h}^{T} P_{h} b_{h}\left(\tilde{\theta}_{\square}^{T} \varphi_{\square}+\tilde{\theta}_{\square}^{T} \varphi \delta\right)-\tilde{\theta}_{\square}^{T} \Gamma_{\square}^{-} \dot{\hat{\theta}}_{\square}- \\
& \sigma_{\square} \tilde{\theta}_{\square f}^{T} \Gamma_{\square f}^{-} \dot{\hat{\theta}}_{\square f}-\tilde{\theta}_{\square}^{T} \Gamma_{\square}^{-} \dot{\hat{\theta}}_{\square}-\sigma \tilde{\theta}_{\square f}^{T} \Gamma_{\square f}^{-\square} \dot{\hat{\theta}}_{\square f} \\
\leq & -e_{h}^{T} Q_{h} e_{h} \square-\sigma_{\square}\left(\tilde{\theta}_{\square f}-\tilde{\theta}_{\square}\right)^{T}\left(\tilde{\theta}_{\square f}-\tilde{\theta}_{\square}\right)- \\
& \sigma_{\square}\left(\tilde{\theta}_{\square f}-\tilde{\theta}_{\square}\right)^{T}\left(\tilde{\theta}_{\square f}-\tilde{\theta}_{\square}\right)
\end{aligned}
$$$$
\leq \square
$$

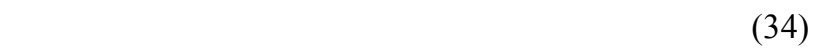

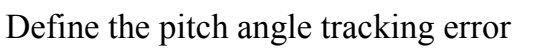

$$
\square
$$

$$
\tilde{\theta}=\theta-\theta_{r e f} \dot{\tilde{\theta}}=q-\dot{\theta}_{r e f} \square
$$

एा1ा

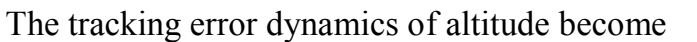

$$
\ddot{\tilde{\theta}}=\theta_{\square}^{T} \varphi_{\square}+\theta_{\square}^{T} \varphi \delta-\ddot{\theta}_{r e f} \square
$$

$\square+11$

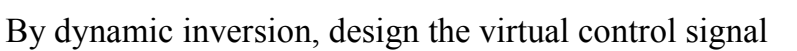

$$
u_{\theta}=\hat{\theta}_{\square}^{T} \varphi \delta=-k_{P \theta} \tilde{\theta}-k_{D \theta} \dot{\tilde{\theta}}-\hat{\theta}_{\square}^{T} \varphi_{\square}+\ddot{\theta}_{r e f}^{\square \square \square \square}
$$

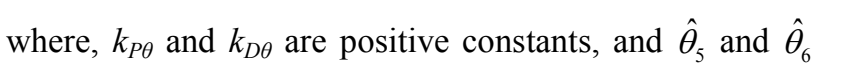

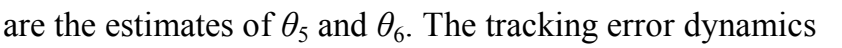
$\square \amalg \square \square$ $\square \quad\left[\begin{array}{c}\dot{\tilde{\theta}} \\ \ddot{\tilde{\theta}}\end{array}\right]=\underbrace{\left[\begin{array}{cc}\square & \square \\ -k_{P \theta} & -k_{D \theta}\end{array}\right]}_{A_{\theta}}\left[\begin{array}{c}\tilde{\theta} \\ \dot{\tilde{\theta}}\end{array}\right]+\left[\begin{array}{c}\square \\ \square\end{array}\right]\left(\tilde{\theta}_{\square}^{T} \varphi_{\square}+\tilde{\theta}_{\square}^{T} \varphi \delta\right) \square \square \square \square$

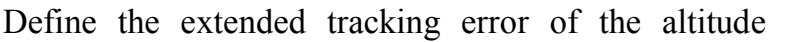
एाषाणमए

$$
e_{\theta}=\tilde{\theta} \dot{\tilde{\theta}} \dot{H}^{T} \square
$$

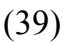

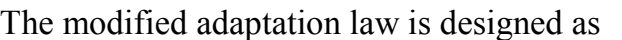

$$
\begin{aligned}
& \dot{\hat{\theta}}_{\square}=\Gamma_{\square} \square \square\left(\varphi e_{\theta}^{T} P b-\sigma_{\square}\left(\hat{\theta}_{\square}-\hat{\theta}_{\square f}\right)\right) \square \square \square \square \\
& \dot{\hat{\theta}}_{\square}=\Gamma_{\square} \square \square\left(\varphi \delta e_{\theta}^{T} P b-\sigma_{\square}\left(\hat{\theta}_{\square}-\hat{\theta}_{\square f}\right)\right) \square \square \square \square
\end{aligned}
$$

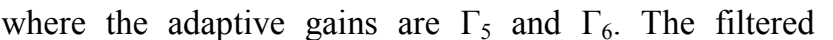

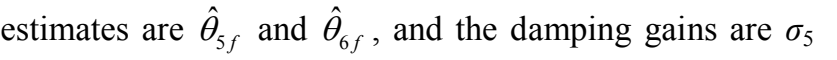

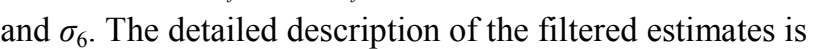

$$
\begin{array}{ll}
\dot{\hat{\theta}}_{\square f}=\Gamma_{\square f}\left(\hat{\theta}_{\square}-\hat{\theta}_{\square f}\right) \square & \square \square \square \\
\dot{\hat{\theta}}_{\square f} & =\Gamma_{\square f}\left(\hat{\theta}_{\square}-\hat{\theta}_{\square f}\right) \square
\end{array}
$$

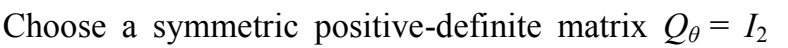

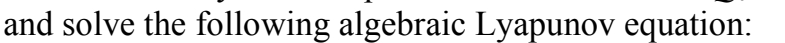

$$
P_{\theta} A_{\theta}+A_{\theta}^{T} P_{\theta}=-Q_{\theta} \square
$$

एा1ए

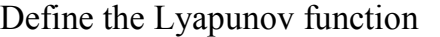

$$
\begin{gathered}
W_{\theta}=e_{\theta}^{T} P_{\theta} e_{\theta} \square+\tilde{\theta}_{\square}^{T} \Gamma_{\square}^{-} \tilde{\theta}_{\square} \square+\left(\sigma_{\square} \square\right) \tilde{\theta}_{\square f}^{T} \Gamma_{\square f}^{-\square} \tilde{\theta}_{\square f}+ \\
\tilde{\theta}_{\square}^{T} \Gamma_{\square}^{-} \tilde{\theta}_{\square} \square+\left(\sigma_{\square} \square\right) \tilde{\theta}_{\square f}^{T} \Gamma_{\square f}^{-\square} \tilde{\theta}_{\square f}
\end{gathered}
$$

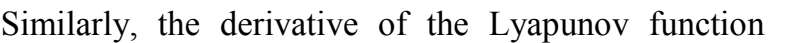

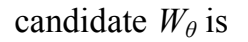

$$
\begin{aligned}
\dot{W}_{\theta} \leq & -e_{\theta}^{T} Q_{\theta} e_{\theta} \square-\sigma_{\square}\left(\tilde{\theta}_{\square f}-\tilde{\theta}_{\square}\right)^{T}\left(\tilde{\theta}_{\square f}-\tilde{\theta}_{\square}\right)- \\
& \sigma_{\square}\left(\tilde{\theta}_{\square f}-\tilde{\theta}_{\square}\right)^{T}\left(\tilde{\theta}_{\square f}-\tilde{\theta}_{\square}\right) \\
\leq & \square
\end{aligned}
$$$$
\text { एIIIII }
$$

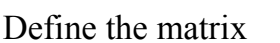

$$
G(x)=\left[\hat{\theta}_{\square}^{T} \varphi_{\square} \hat{\theta}_{\square}^{T} \varphi_{\square}\right]^{T} \square
$$

밈

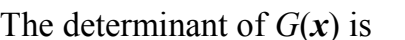

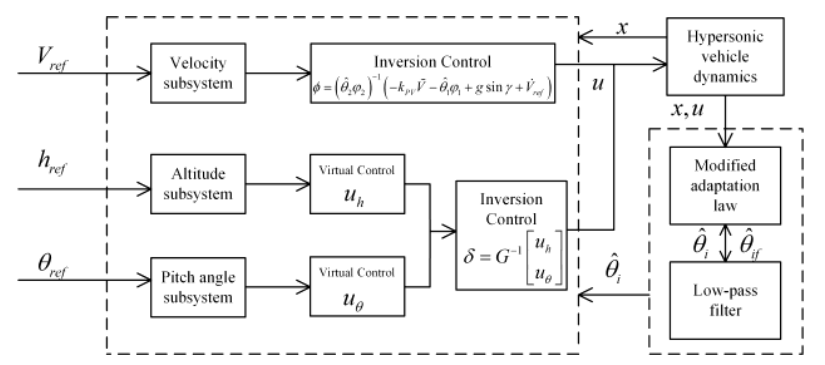

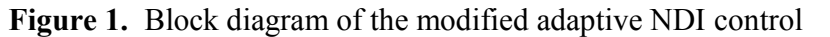
$\square|\|| 1 \mid \square$ 


$$
\begin{aligned}
\square(G(x))= & V \square \gamma \bar{q} S \bar{c} . \\
& \left(v_{\square} v_{\square} C_{M}^{\delta_{c}} C_{L}^{\delta_{e}}-v_{\amalg} v_{\amalg} C_{M}^{\delta_{e}} C_{L}^{\delta_{c}}\right) \square \square \square \square \\
& \left(v_{\amalg} v_{\square} m I_{y y}\right)
\end{aligned}
$$

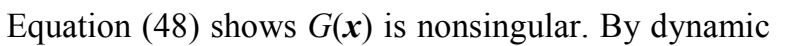

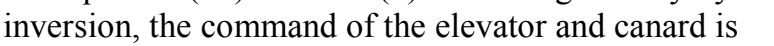

$$
\boldsymbol{\delta}=G^{-\square}\left[u_{h} \boldsymbol{u}_{\theta}\right]^{T} \square
$$

$\llbracket 11$

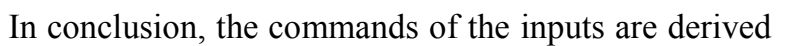

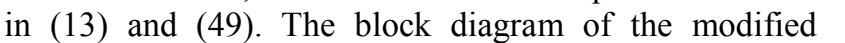

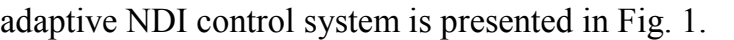

\subsection{Pole placement technique}

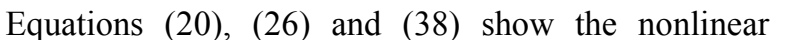

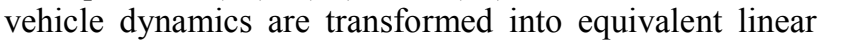

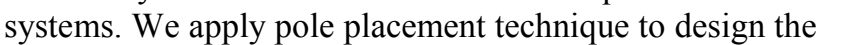

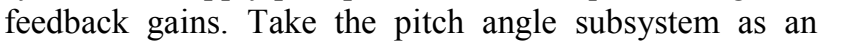

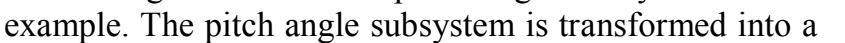

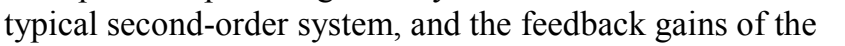

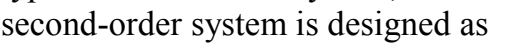

$$
\square
$$

$$
k_{P \theta}=\omega_{n}^{\square} k_{D \theta}=\zeta_{n} \omega_{n} \square
$$

$\square+1$

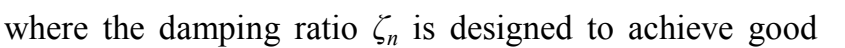

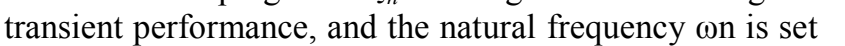

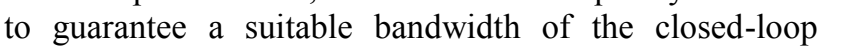
पणापा

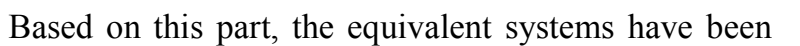

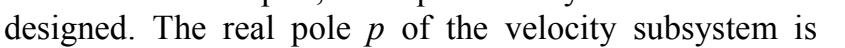

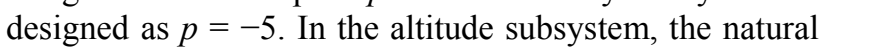

\begin{tabular}{|c|c|c|}
\hline 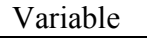 & 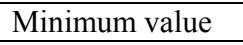 & 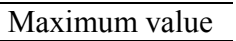 \\
\hline 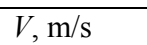 & $\overline{1111 \square}$ & 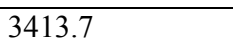 \\
\hline$h \mathbb{\mathrm { m }} \square$ & $\square 11$ & $\square 11$ \\
\hline$\gamma$ पाणा & 西 & $\square$ \\
\hline 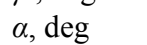 & 西 & $\square$ \\
\hline$Q$ एणामा & एण & $\square$ \\
\hline$\delta$ एाणा & एण & $\square$ \\
\hline$\phi$ & वाणा & पाण \\
\hline
\end{tabular}

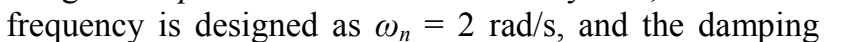

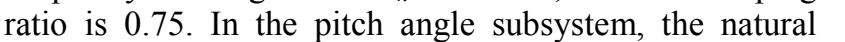

\begin{tabular}{|c|c|c|c|}
\hline 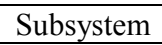 & $\mathrm{\square Im}$ & 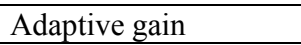 & 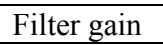 \\
\hline & & $\Gamma_{\square} \square\left[I_{\square} \amalg \sigma_{\square} \square\|\| \square\right.$ & $\overline{\Gamma_{f f} \| \square I}$ \\
\hline பாயாயப & $k_{p V} \amalg \amalg$ & 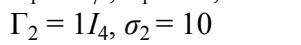 & $\Gamma_{f f}^{\prime} \square I_{\square} \square$ \\
\hline 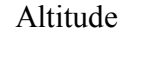 & $k_{p h}$ ШाW & 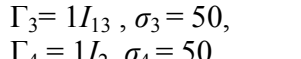 & $\Gamma_{f} \square \square I_{\square \square}$ \\
\hline 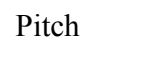 & 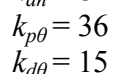 & 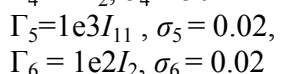 & $\begin{array}{l}\Gamma_{\vdash f} \square \square I_{\square \square} \\
\Gamma_{f} \square \square I \square \square\end{array}$ \\
\hline
\end{tabular}

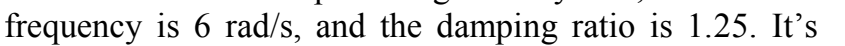

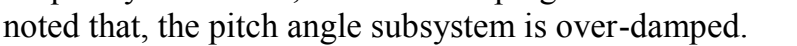

Table 2.

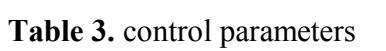

\section{Simulation analysis}

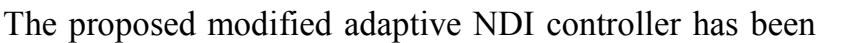

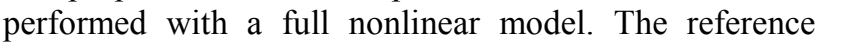

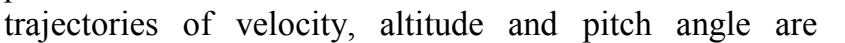

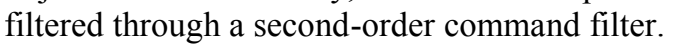

$$
\begin{aligned}
& V_{\square \square} V_{c}=\square \square \square\left(s^{\square}+\square \times \square \square \times \square \square s+\square \square\right) \\
& h_{\square \square} h_{c}=\square \square \square\left(s^{\square}+\square \times \square \square \times \square \square s+\square \square\right) \\
& \theta_{\square \square} \theta_{c}=\square\left(s^{\square}+\square \times \square \times \square+\square\right)
\end{aligned}
$$

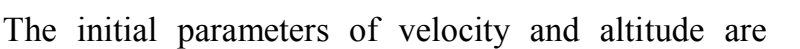

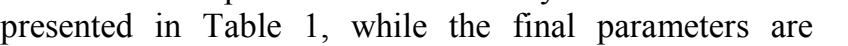

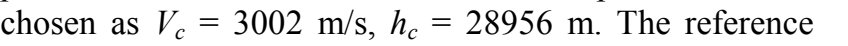

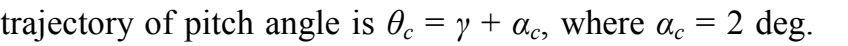

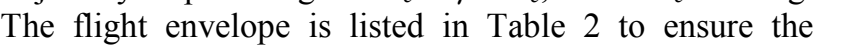

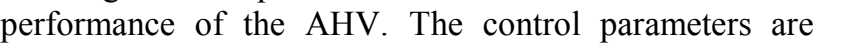

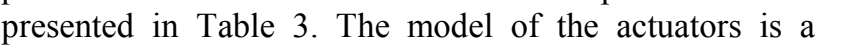

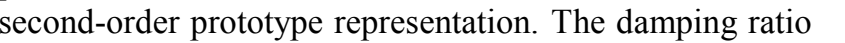

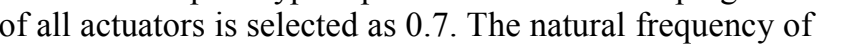

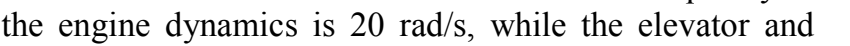

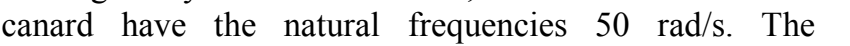

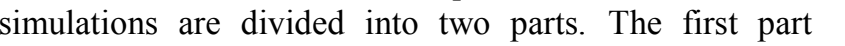

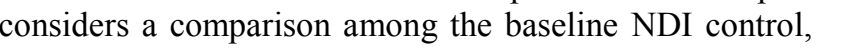

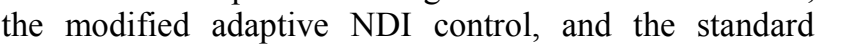

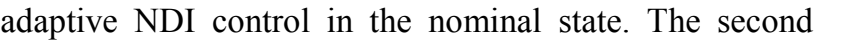

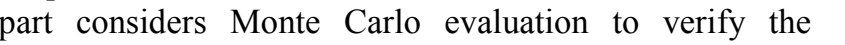

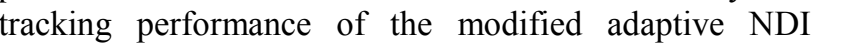

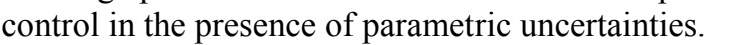

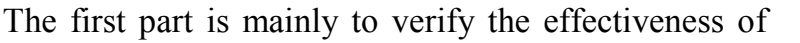

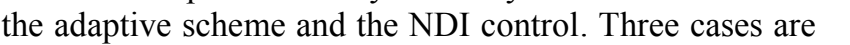

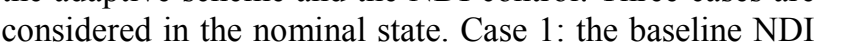

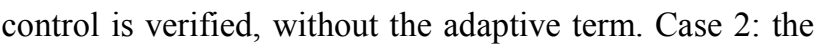

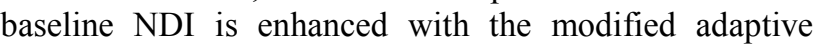

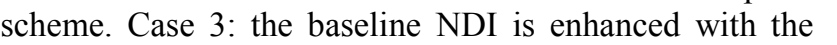

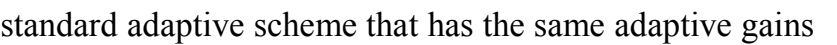

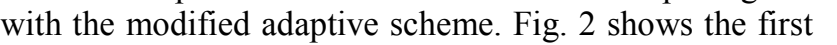

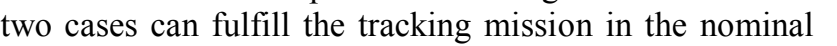

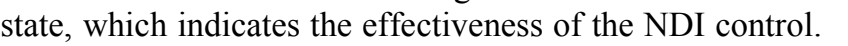

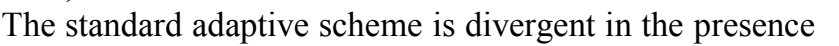

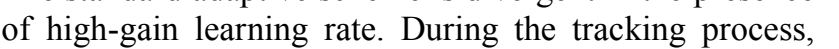

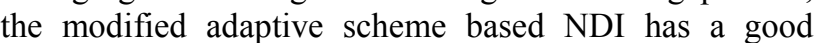

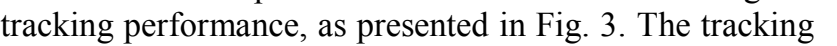

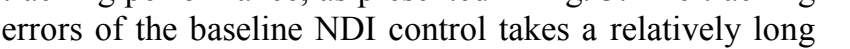

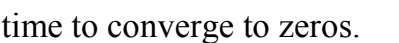

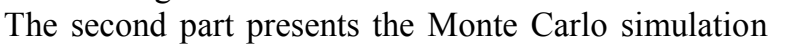

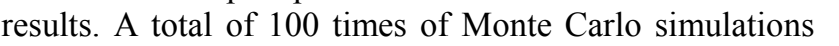

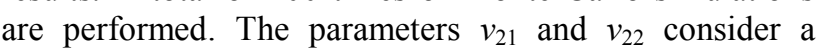

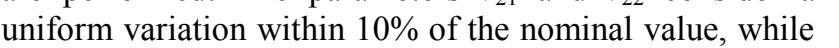

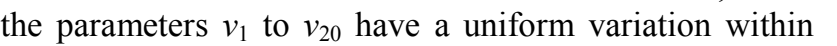

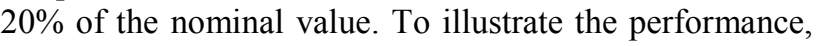

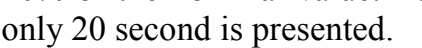
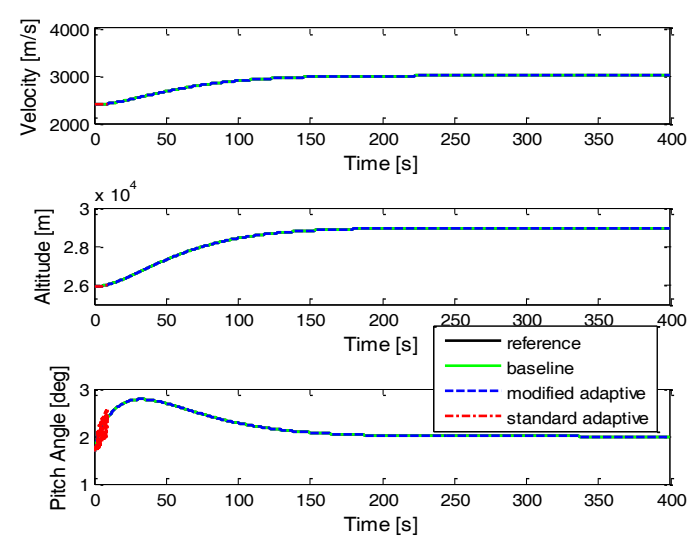

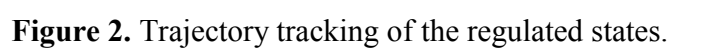




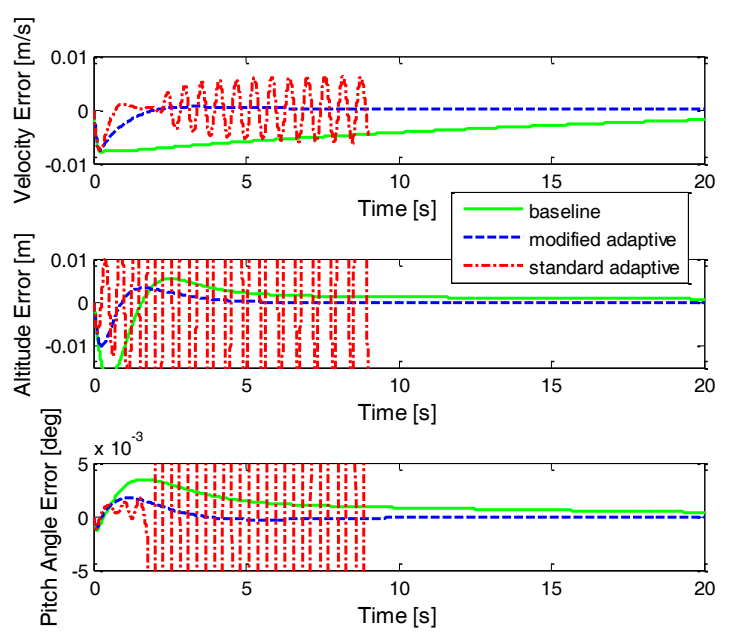

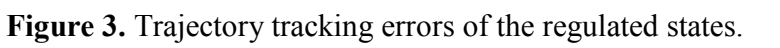
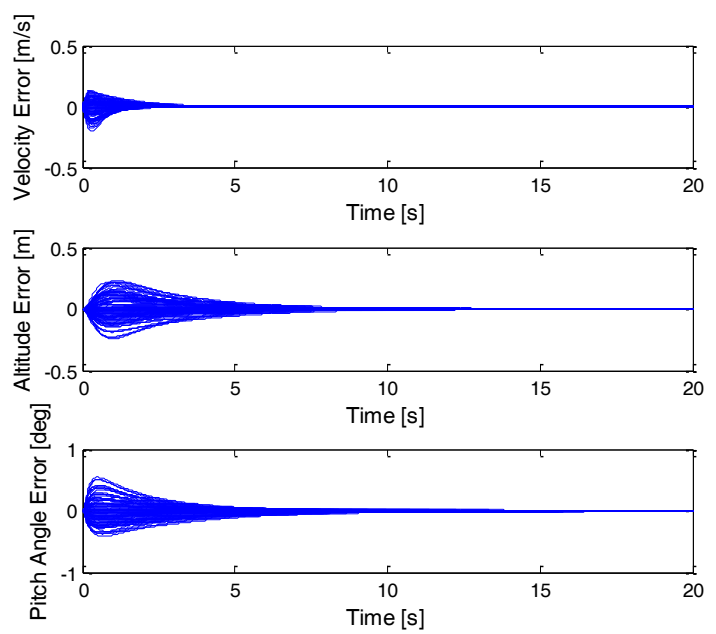

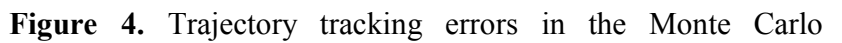
पापाणाणाण
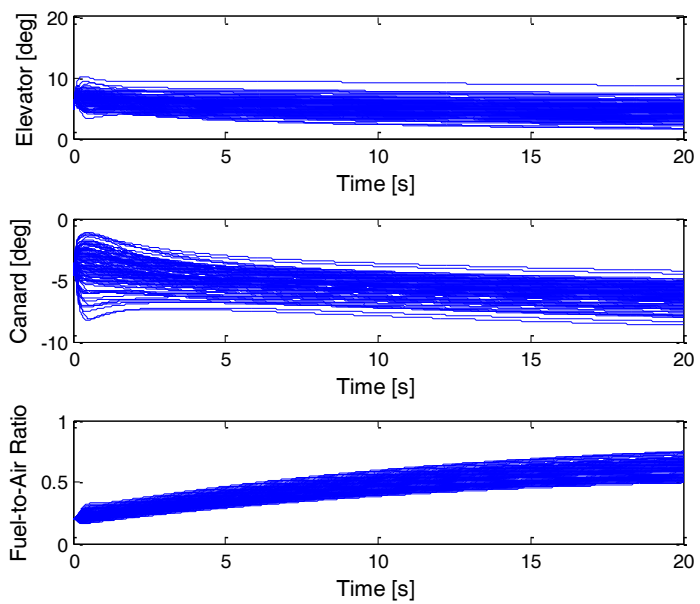

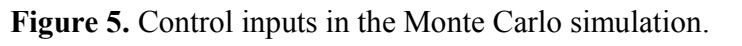

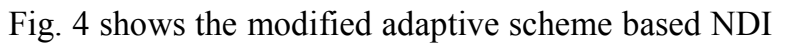

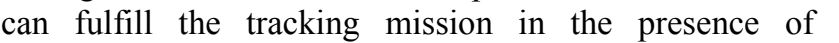

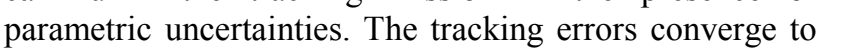

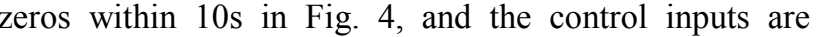

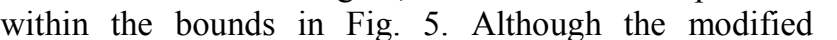

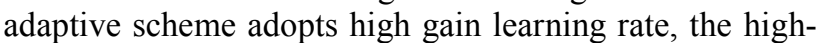

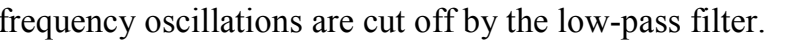

\section{Conclusion}

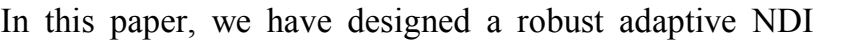

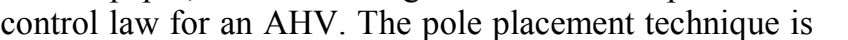

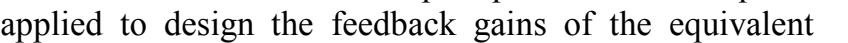

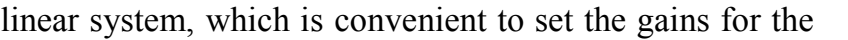

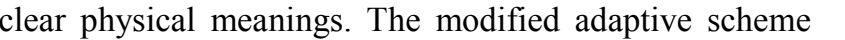

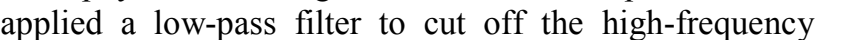

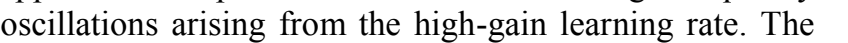

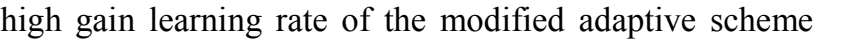

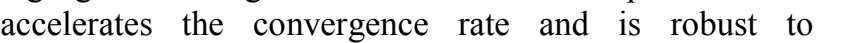

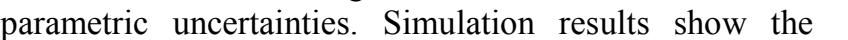

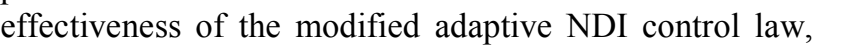

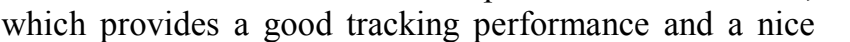

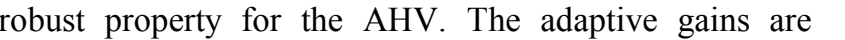

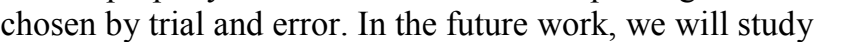

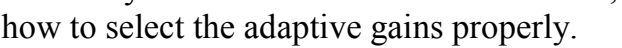

\section{References}

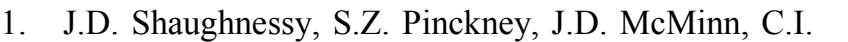

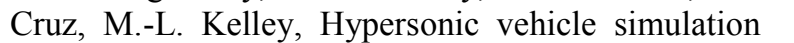

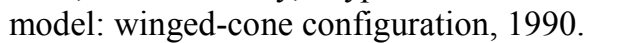

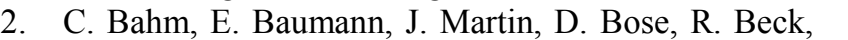

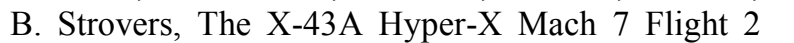

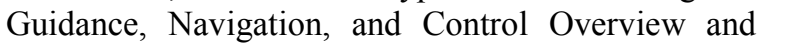

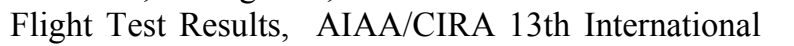

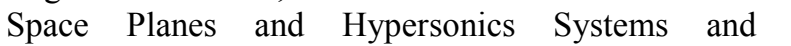

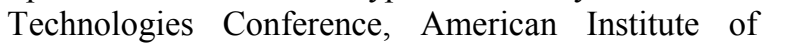

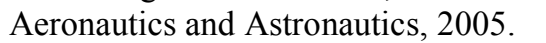

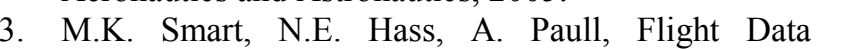

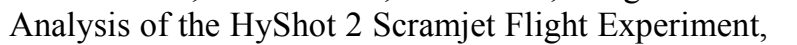

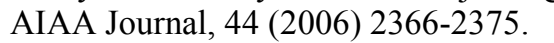

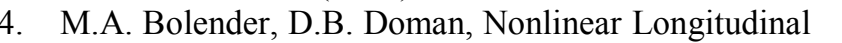

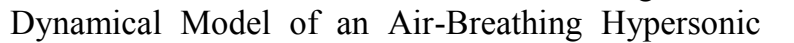

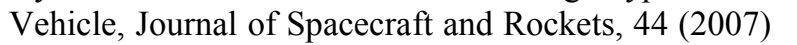
पाणापाप

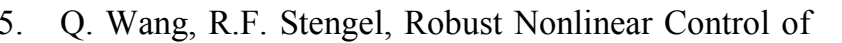

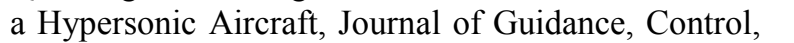

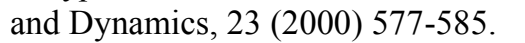

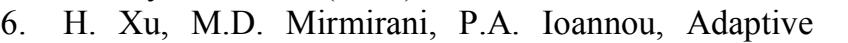

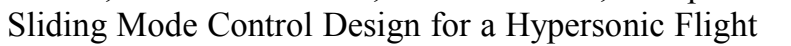

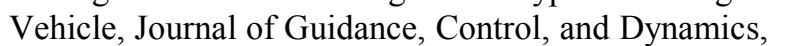

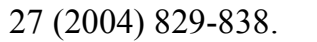

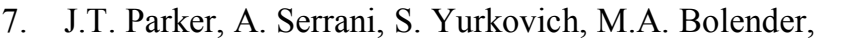

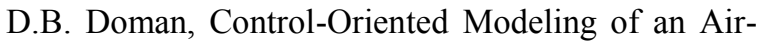

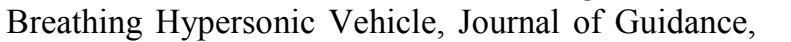

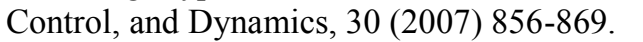

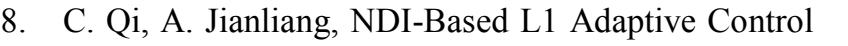

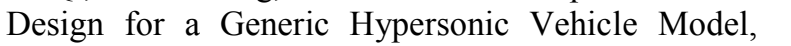

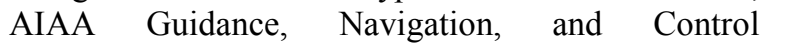

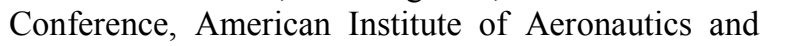

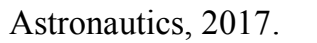

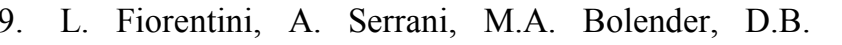

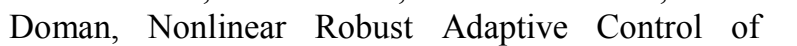

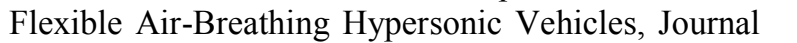




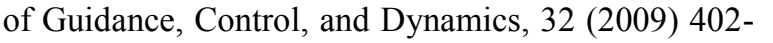
$\square 1 \mathrm{ll}$

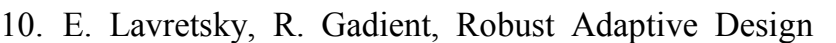

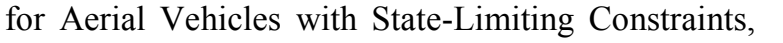

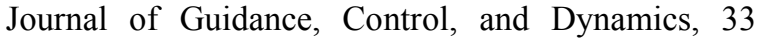

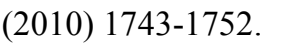

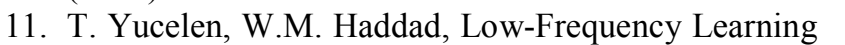

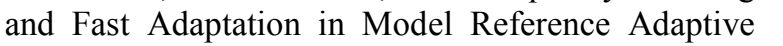

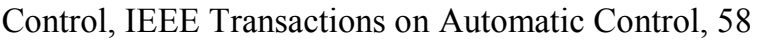
एमाएणामामाण

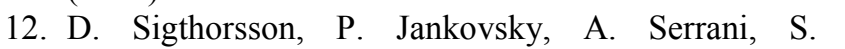

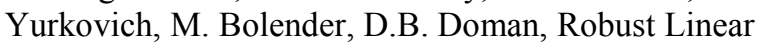

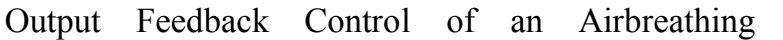

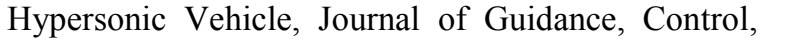

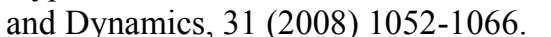

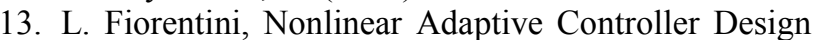

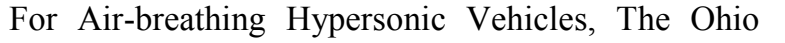

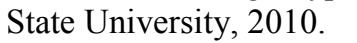

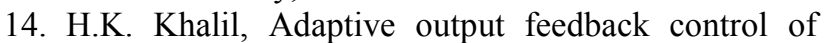

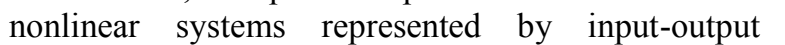

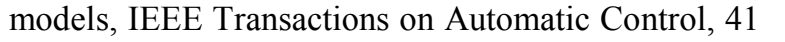

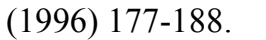

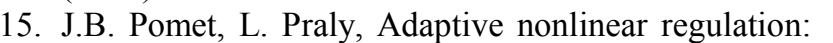

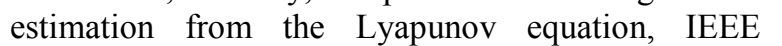

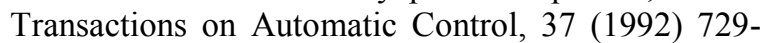
$\square \square$ 